\title{
Serum substance $P$ levels in patients with chronic schizophrenia treated with typical or atypical antipsychotics
}

\author{
Takahiko Nagamine \\ Division of Psychiatric Internal \\ Medicine, Seiwakai-Kitsunan Hospital \\ 338I Suzenji, Yamaguchi City, Japan
}

Correspondence:Takahiko Nagamine Division of Psychiatric Internal Medicine, Seiwakai-Kitsunan Hospital 338I Suzenji, Yamaguchi-City, Yamaguchi-Pref. 747-I22I, Japan

Tel +8I 08398621 II

Fax +8I 0839863076

Email anagamine@ybb.ne.jp

\begin{abstract}
Aspiration pneumonia is a major cause of death in patients with dysphagia, often accompanied by psychiatric symptoms. The inhibition of swallowing and cough reflexes, which contribute to a significant risk for aspiration, may be related to decreased levels of substance P. Clinical studies indicate a strong association of an increased mortality in psychiatric patients with the use of antipsychotics. The present study documented fewer positive episodes of swallowing reflex in patients treated with haloperidol for schizophrenia $(7 / 11 ; 63.6 \%)$ than those treated with risperidone $(10 / 11 ; 90.9 \%)$. In addition, patients treated with risperidone had serum substance P levels comparable with control subjects $(29.0 \pm 7.8 \mathrm{pg} / \mathrm{mL}, 29.6 \pm 7.6$, respectively; $\mathrm{p}=0.9$ ), while patients treated with haloperidol had significantly lower serum substance $P$ levels $(20.6 \pm 5.5 \mathrm{pg} / \mathrm{mL} ; \mathrm{p}<0.01)$. Among patients on haloperidol, those with negative episodes of reflex $(4 / 11 ; 36.4 \%)$ had serum substance P levels at $15.8 \pm 1.0 \mathrm{pg} / \mathrm{mL}$, in contrast with those with positive episodes $(7 / 11 ; 63.6 \%)$ who had serum levels at $23.4 \pm 4.9 \mathrm{pg} / \mathrm{mL}$. However, in the patient group treated with risperidone, serum substance $\mathrm{P}$ levels in the majority of patients with positive episodes of reflexes $(10 / 11,90.9 \% ; 30.1 \pm 7.2 \mathrm{pg} / \mathrm{mL})$ was found to be as high as in control subjects, all with positive episodes $(5 / 5,100 \% ; 29.6 \pm 7.6 \mathrm{pg} / \mathrm{mL})(\mathrm{p}=0.866)$, and higher than in one patient with negative reflex $(1 / 11,9.1 \% ; 18.0 \pm 0.0 \mathrm{pg} / \mathrm{mL})$. These results suggest that the decreased serum substance P levels are strongly associated with the use of haloperidol, as well as decreased swallowing reflexes. This suggests that serum substance $\mathrm{P}$ levels may be a useful predictive marker for the increased risk of developing aspiration, or subsequently aspiration pneumonia. Moreover, this increased incidence of aspiration may contribute to an increased mortality in patients following antipsychotic therapy. Risperidone, which has little influence on serum substance $\mathrm{P}$ productions, may be a more appropriate first-line drug of choice for treatment of schizophrenia
\end{abstract}

Keywords: schizophrenia, aspiration pneumonia, substance P, risperidone, haloperidol

\section{Introduction}

Aspiration pneumonia is a major cause of death in elderly patients over 60 years old, especially with dysphagia, often accompanied by psychiatric or underlying causes (Haleem 1990; Elpern 1997; Fioritti et al 1997). It has recently been shown that the use of antipsychotic drugs is linked to increased risk of death among the elderly patients with dementia and neuropsychiatric symptoms (Schneider et al 2005; Rabins and Lyketsos 2005; Wang et al 2005). Moreover, retrospective analyses suggest that typical antipsychotics seemed to be associated with a higher risk for death than are atypical antipsychotics (Nasrallah et al 2004; Wang et al 2005). Deaths in these clinical trials seem to have been primarily related to cardiac arrhythmias and aspiration pneumonia (Pontoppidant and Beecher 1960; Nakagawa et al 1993; FDA 2006). The majority of patients who aspirate have clinically silent aspiration with no recognizable signs or symptoms (Linden and Siebens 1983; Pikus et al 2003). Silent aspiration remains 
a major clinical problem, because the clinical history is not beneficial in determining patients at a risk for the development of aspiration pneumonia (Logemann 1983; Pikus et al 2003). In fact, it has been shown that silent aspiration associated with impaired swallowing and cough reflexes in the elderly leads to a high risk of developing aspiration pneumonia (Horner and Massey 1988; Holas et al 1994). In other word, the risk of developing aspiration pneumonia may be substantially higher in patients with silent aspiration compared with those in whom aspiration elicits reflexes. Therefore, the use of antipsychotic medications in elderly patients may increase development of silent aspiration, or aspiration pneumonia leading to an increased risk of mortality in this patient group.

Substance $\mathrm{P}$ has been shown to be the natural endogenous ligand of tachykinin NK1 receptors that control the swallowing and cough reflexes (Regoli et al 1994; Yamaya et al 2001; Zachrisson et al 2000). Tachykinins which act on NK receptors have implications for the clinical use of NK receptor antagonists in schizophrenia (Chahl 2006). Administration of haloperidol significantly decreases substance P-encoding mRNAs, suggesting a drug-induced decrease in nigrostriatal tachykinin biosynthesis (Bannon et al 1986). Thus, the use of haloperidol might contribute to a lowering of serum substance $\mathrm{P}$ levels in psychotic patients, and this depression of substance $\mathrm{P}$ production might be a major risk for developing aspiration in the elderly because of the associated impaired swallowing and cough reflex with senescence. Taking these results into consideration, it is hypothesized that the increased risk of death in elderly patients, associated with the use of antipsychotics and the resulting decrease in substance P levels, might be attributable to the increased risk of developing silent aspiration, or potentially aspiration pneumonia.

The present study was undertaken to examine the relationship of serum substance $P$ levels with antipsychotic drug use and with the risk of developing aspiration, or aspiration pneumonia in patients with chronic schizophrenia.

\section{Methods}

\section{Subjects}

The Ethical Committee of Seiwakai-Kitsunan Hospital reviewed and approved this study protocol. All participants gave their written informed consent. The investigations were performed according to the principles expressed in the Declaration of Helsinki. Twenty-two patients, 49-74 year-old, both males and females, and diagnosed with schizophrenia by DMS-IV criteria, were treated with risperidone or haloperidol monotherapy for a minimum of 1 year. Five demographically matched control subjects were included in this study. The exclusion criteria were (1) cerebral infarction (verified by X-ray computed tomographic scan of the brain) and (2) bronchial asthma.

\section{Measurements of serum substance $P$ Isolation of serum samples}

The peripheral blood samples were mixed with EDTA-2Na immediately after drawn, followed by the addition of aprotinin to avoid degradation of substance $P$. Samples were then centrifuged at $4{ }^{\circ} \mathrm{C}$. The resulting plasma was stored at $-20^{\circ} \mathrm{C}$ until assay. An aliquot of $0.5 \mathrm{~mL}$ plasma sample was mixed with $1 \mathrm{~mL}$ acetone and then centrifuged at $3000 \mathrm{rpm}$ at $4{ }^{\circ} \mathrm{C}$ for 10 minutes. The supernatant was delipidized with $1 \mathrm{~mL}$ petroleum ether and the upper layer was discarded. This manipulation was executed twice. The lower layer was evaporated by nitrogen gas at $45^{\circ} \mathrm{C}$ and reconstituted with $0.25 \mathrm{~mL}$ assay buffer, which was provided for serum samples.

\section{Measurements of serum substance $P$ by radioimmunoassay}

Petroleum ether extract $0.2 \mathrm{~mL}$ or standard substance P were mixed with anti-substance $\mathrm{P}$ antibody, and incubated at $4{ }^{\circ} \mathrm{C}$ overnight. Samples were then incubated with ${ }^{125} \mathrm{I}_{\text {-tyr }}{ }^{8}$-substance $\mathrm{P}$ at $4{ }^{\circ} \mathrm{C}$ for $4 \mathrm{~h}$. The mixture was mixed with $1 \% \gamma$-globulin and $17.5 \%$ polyethylene glycol, and then centrifuged at $3000 \mathrm{rpm}$ at $4{ }^{\circ} \mathrm{C}$ for 20 minutes. The supernatant was discarded, and the radioactivity of the pellets was measured by a $\gamma$-counter.

\section{Simple swallowing provocation test}

A two-step simple swallowing provocation test (SSPT; Teramoto et al 1999) was conducted as a means of assessing the aspiration status. A $3 \mathrm{Fr}$ feeding tube was inserted to the uvula from the nose in a lateral position, and infused once with $2 \mathrm{ml}$ of distilled water. If a swallowing reaction occurred within 3 seconds, the swallowing reflex was judged as positively induced (p-reflex), and if it did not occur, the swallowing reflex was judged as negatively induced (n-reflex).

\section{Statistical methods}

Groups were compared using an unpaired t-test, and $\chi^{2}$-test where applicable with $\mathrm{p}<0.05$ indicating a significant difference. The values were expressed as the mean \pm SD.

\section{Results}

\section{Characteristics of subjects}

Table shows the basic characteristics of the 22 schizophrenic patients who received typical (haloperidol, $\mathrm{n}=11 ; 7$ male 
and 4 female) or atypical (risperidone, $\mathrm{n}=11 ; 6$ male and 5 male) antipsychotic drugs. Patients were being treated with risperidone or haloperidol, at a mean dosage of 536.3, or 552.2 mg chlorpromazine per day each, the range going from 3.2 to $7.6 \mathrm{mg}$ /day (mean $5.4 \mathrm{mg}$ ), or from 4.5 to $15.7 \mathrm{mg}$ per day (mean $10.1 \mathrm{mg}$ ) each. Control subjects consisted of 5 healthy adults, aged 38 to 56 years of age (mean 46.2 years; 2 male and 3 female). Age, disease duration and BMI were similar in each group; no significant differences were seen among three groups in characteristics. The mean chlorpromazineequivalent dosages administered to the risperidone and haloperidol groups were similar. However, schizophrenia patients receiving haloperidol have a tendency to have lesser positive episodes of swallowing $(7 / 11 ; 63.6 \%)$ compared to those on risperidone $(10 / 11 ; 90.9 \%)$ (Table 1).

\section{Association of serum substance $P$ levels with antipsychotic drug use and swallowing reflex}

A possible associations of serum substance $P$ levels with different classes of antipsychotic drugs used for treatment were examined, as well as, relationships to episodes of swallowing reflex using SSPT in patients with schizophrenia. As shown in Figure 1, the mean substance P levels were significantly lower in the haloperidol group $(20.6 \pm 5.5 \mathrm{pg} / \mathrm{mL})$ when compared to the risperidone group $(29.0 \pm 7.8 \mathrm{pg} / \mathrm{mL})(\mathrm{p}<0.01)$. Substance P levels for the control subjects $(29.6 \pm 7.6 \mathrm{pg} / \mathrm{mL})$ was not significantly different than the risperidone group $(p=0.8878)$. No correlation was found for either age or sex with serum substance $\mathrm{P}$ levels $(\mathrm{r}=0.2)$.

The relationship between the induction of swallowing reflex and serum substance $P$ levels in all patients receiving either haloperidol or risperidone is shown in Figure 2. When substance $\mathrm{P}$ levels were correlated in all patients based upon episodes of reflex, higher substance $\mathrm{P}$ levels were seen in patients with positive episodes of reflex (swallowing reflex elicited, p-Reflex; $27.4 \pm 7.1 \mathrm{pg} / \mathrm{mL}$ ) than in patients with negative reflex (swallowing reflex not elicited, n-Reflex; $16.2 \pm 1.3 \mathrm{pg} / \mathrm{mL})(\mathrm{p}<0.001)$.

Substance $P$ levels were evaluated in patients treated with either haloperidol or risperidone and grouped according to episodes of reflex. Among patients on haloperidol, higher substance $P$ levels were seen in patients with positive episodes of reflex $(23.4 \pm 4.9 \mathrm{pg} / \mathrm{mL})$ than in patients with negative episodes of reflex $(15.8 \pm 1.0 \mathrm{pg} / \mathrm{mL})(\mathrm{p}<0.01)$ (Figure 3), showing generally lower substance P levels in patients on haloperidol, with either positive or negative episodes, when compared to patients on risperidone or control subjects. In contrast, in the risperidone group, serum substance $\mathrm{P}$ levels in the majority of patients with positive episodes of reflexes $(10 / 11,90.9 \% ; 30.1 \pm 7.2 \mathrm{pg} / \mathrm{mL})$ was found as high as in control subjects all with positive episodes $(5 / 5,100 \% ; 29.6 \pm 7.1 \mathrm{pg} / \mathrm{mL})$, and higher than in one patient with negative reflex $(1 / 11,9.1 \% ; 18.0 \pm 0.0 \mathrm{pg} / \mathrm{mL} ; \mathrm{p}<0.01)$ (Figure 3). These results indicate a strong association of substance $\mathrm{P}$ levels with episodes of reflex in patients with schizophrenia.

When the association of different classes of antipsychotic drug use with episodes of reflex was examined, positive episodes of reflex were higher in patients receiving risperidone $(90.9 \% ; 10 / 11)$ than that in patients receiving haloperidol $(63.6 \% ; 7 / 11)$ (Table 1). It is notable that negative episodes of reflex in patients receiving haloperidol $(4 / 11 ; 36.4 \%)$ were 4 times as high as that on risperidone (1/11;9.1\%). In addition, in the haloperidol treated subjects, substance P levels, even in positive episodes, were found to be relatively lower (23.4 $\pm 4.9 \mathrm{pg} / \mathrm{mL})$ than either the risperidone $(29.0 \pm 7.8 \mathrm{pg} / \mathrm{mL})$, or on control subjects $(29.6 \pm 7.6 \mathrm{pg} / \mathrm{mL})$.

Table I Baseline characteristics and outcome measures

\begin{tabular}{|c|c|c|c|c|}
\hline & $\begin{array}{l}\text { Haloperidol } \\
\mathbf{N}=\text { I I }\end{array}$ & $\begin{array}{l}\text { Risperidone } \\
\mathbf{N}=\text { I I }\end{array}$ & $\begin{array}{l}\text { Control } \\
\mathbf{N}=\mathbf{5}\end{array}$ & p-value \\
\hline Age (mean $\pm S D$ years) & $58.4 \pm 6.3$ & $57.5 \pm 8.7$ & $46.2 \pm 6.5$ & n.s.* \\
\hline Gender: Male/female & $7 / 4$ & $6 / 5$ & $2 / 3$ & \\
\hline Body mass index & $22.0 \pm 3.1$ & $21.9 \pm 2.7$ & $22.2 \pm 1.8$ & n.s. \\
\hline Age at illness onset (years) & $23.7 \pm 3.8$ & $24.4 \pm 3.7$ & $N / A^{* *}$ & n.s. \\
\hline Duration of treatment (month) & $25.9 \pm 7.7$ & $23.3 \pm 7.1$ & N/A & n.s. \\
\hline Dose (mg/day) & $10.1 \pm 5.6$ & $5.4 \pm 2.2$ & $\mathrm{~N} / \mathrm{A}$ & \\
\hline Chlorpromazine equiv (mg/day) & $552.2 \pm 317.1$ & $536.3 \pm 220.3$ & N/A & n.s. \\
\hline Serum substance $P(p g / m l)$ & $20.6 \pm 5.5$ & $29.0 \pm 7.8$ & $29.6 \pm 7.6$ & $\mathrm{p}<0.01$ \\
\hline Positive episodes of swallowing reflex & $7 / 11$ (63.6\%) & $10 / 11$ (90.9\%) & $5 / 5(100 \%)$ & n.s. \\
\hline
\end{tabular}

Notes: *Not significant, ${ }^{*}$ Not applicable 


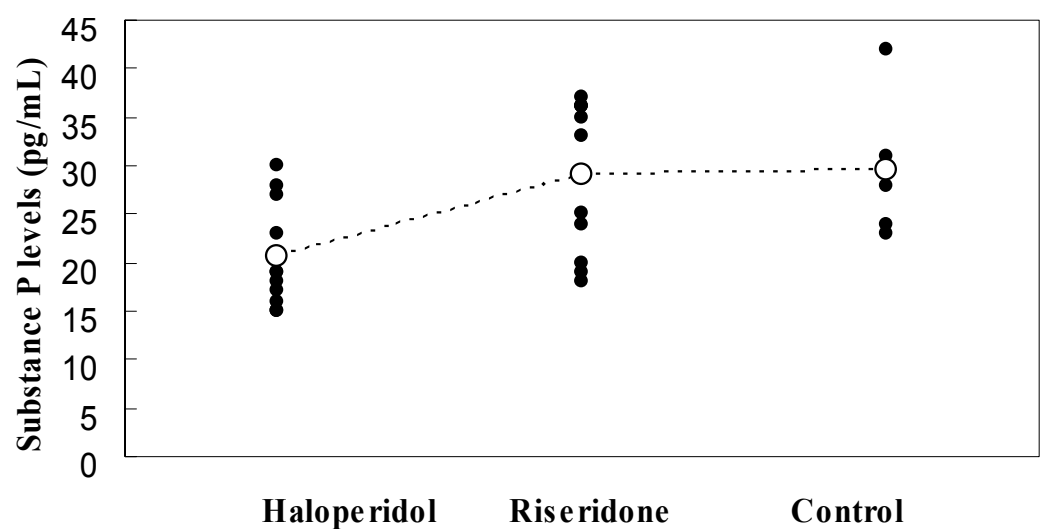

Figure I Serum substance P levels in antipsychotics-treated patients with schizophrenia and untreated control subjects. Serum substance P levels were measured for patients on haloperidol or risperidone, and for control subjects. Horizontal bars represent average levels of serum substance P. Dots represent levels in each individual patient.

These results suggest that lower serum substance $P$ levels are evoked by the use of haloperidol, resulting in reduction of reflex. Thus, it appears likely that a low substance $\mathrm{P}$ levels may potentially contribute to a higher risk of developing aspiration in patients with schizophrenia, which may in turn lead to increased mortality in patients after antipsychotic therapy.

\section{Discussion}

The present study has shown that patients on haloperidol, over a period of a 14-37 months, were less likely to demonstrate positive episodes of swallowing reflex $(7 / 11 ; 63.6 \%)$ than those on risperidone $(10 / 11 ; 90.9 \%)$ (Table 1). Treatment with haloperidol resulted in lower serum substance P levels
$(20.6 \pm 5.5 \mathrm{pg} / \mathrm{mL})$ than on risperidone $(29.0 \pm 7.8 \mathrm{pg} / \mathrm{mL}$; $\mathrm{p}<0.01$ ), which was comparable to untreated, control subjects $(29.6 \pm 7.6 \mathrm{pg} / \mathrm{mL} ; \mathrm{p}=0.9)$. Among patients on haloperidol, those with negative episodes of reflex (4/11; $36.4 \%$ ) had serum substance P levels at $15.8 \pm 1.0 \mathrm{pg} / \mathrm{mL}$ in contrast with those with positive episodes $(7 / 11 ; 63.6 \%)$ who had levels at $23.4 \pm 4.9 \mathrm{pg} / \mathrm{mL}(\mathrm{p}<0.01)$. In contrast, in the risperidone treatment group, serum substance $\mathrm{P}$ levels in the majority of patients with positive episodes of reflex $(10 / 11,90.9 \% ; 30.1 \pm 7.2 \mathrm{pg} / \mathrm{mL})$ was found to be as high as in control subjects all with positive episodes $(5 / 5,100 \%$; $29.6 \pm 7.6 \mathrm{pg} / \mathrm{mL})(\mathrm{p}=0.866)$, and higher than in one patient with negative reflex $(1 / 11,9.1 \% ; 18.0 \pm 0.0 \mathrm{pg} / \mathrm{mL})$. These results strongly suggest that the use of haloperidol, which

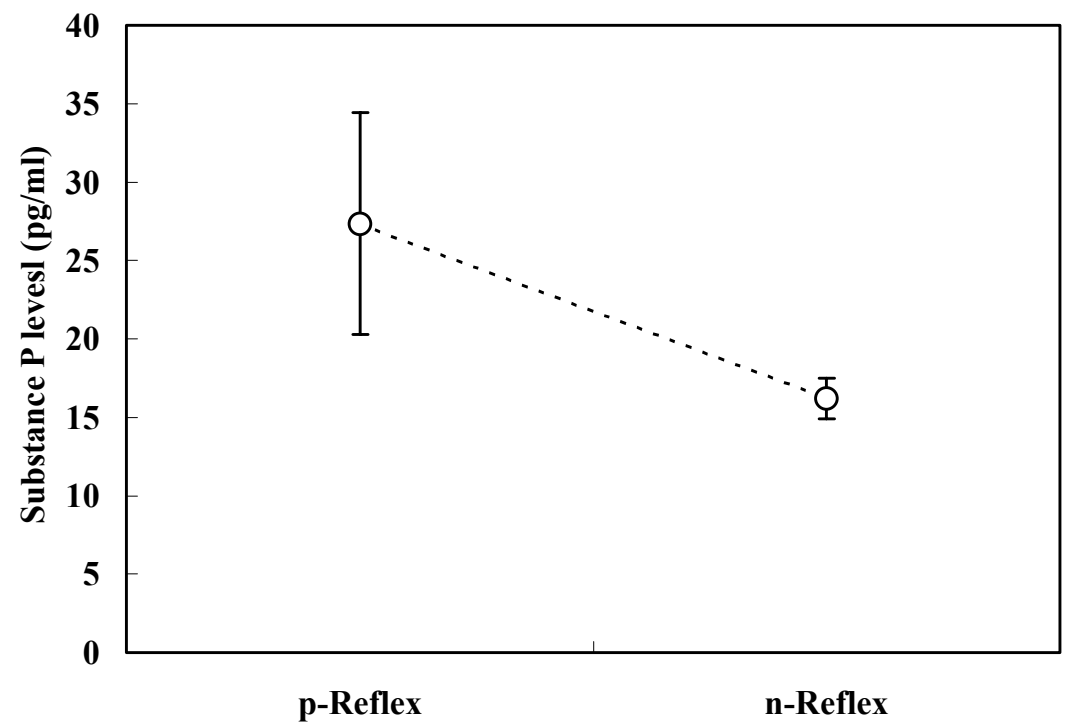

Figure 2 Serum substance $\mathrm{P}$ levels in all patients with or without episodes of reflexes. Substance P levels were measured for patients grouped as: with episodes ( $\mathrm{p}$-reflex) or without episodes of reflexes ( $\mathrm{n}$-reflex). Vertical bars indicate SD. 


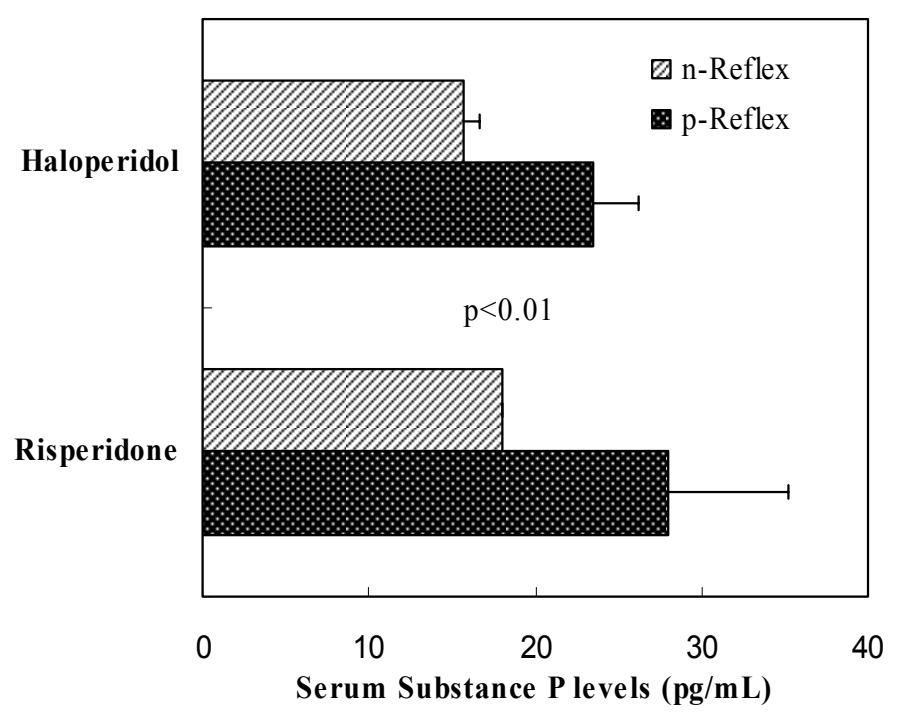

Figure 3 Substance P levels in patients with or without episodes of reflexes. Substance P levels were measured according to: with or without episodes of reflexes on either haloperidol or risperidone.

has a potential action of lowering serum substance $\mathrm{P}$ levels is also associated with lower episodes of swallowing reflex. The likelihood of developing aspiration pneumonia is directly related to the degree of swallowing dysfunctions (Horner and Massey 1988; Nakazawa et al 1993; Holas et al 1994). The review analyses have shown that typical antipsychotic drugs were associated with a significantly higher risk of death than were atypical antipsychotic drugs in elderly psychotic patients at all intervals studied (Schneider et al 2005; Wang et al 2005). Death mostly results from cardiovascular or pneumonia causes (Pontoppidan and Beecher 1960; Nakazawa et al 1993). Thus, it seems likely that haloperidol increases a risk of developing aspiration, or subsequently aspiration pneumonia in schizophrenic patients.

The present study shows a strong association of serum substance P levels with episodes of swallowing reflex. Human studies have shown that swallowing and cough reflexes are governed by a plexus of nerves containing the hormone called substance P (Pernow 1983; Jin 1994). Experimental reduction of substance $\mathrm{P}$ using dopamine antagonists impairs swallowing (Jin 1994), and patients with impaired substance $\mathrm{P}$ production have delayed swallowing reflexes and a higher risk of pneumonia (Nakazawa et al 1997). Thus, as shown in this study, the failure to induce the swallowing reflex using the SSPT in schizophrenic patients appears to have been strongly attributable to a reduction in substance $\mathrm{P}$ production.

As many as $40 \%$ of patients who aspirate have clinically silent aspiration with no signs or symptoms, indicating that the clinical history is often unreliable for determining patients at risk for developing aspiration pneumonia (Linden and Siebens 1983; Pikus et al 2003). Clinical studies have shown that the risk of developing pneumonia is substantially higher in patients with silent aspiration compared with those in whom aspiration elicits cough and swallowing reflexes (Horner and Massey 1988; Holas et al 1994). It is speculative that serum substance $P$ levels may be a useful predictive marker for swallowing difficulties and for the increased incidence of developing silent aspiration, potentially resulting in aspiration pneumonia in elderly patients. Alternatively, this increased incidence of silent aspiration may be responsible for increased mortality in schizophrenic patients using antipsychotic drugs.

Although the present study does not provide any causal relationship between swallowing reflex and substance $P$ levels, it has been shown that administration of haloperidol significantly decreases substance P-encoding mRNAs, suggesting a drug-induced decrease in nigrostriatal tachykinin biosynthesis (Bannon et al 1986). The present study, combined with these results, suggests that the use of haloperidol leads to decreased production of substance $P$, perhaps due to impaired striatal-nigral receptors, and is associated with lower levels of swallowing reflexes.

In conclusion, due to the control that substance $\mathrm{P}$ has on the swallowing and cough reflexes, patients on haloperidol are at an increased risk of developing aspiration or subsequently aspiration pneumonia. In particular, silent aspiration may significantly increase the risk of death in this patient group. During treatment with typical antipsychotic drugs, a reduction of substance $\mathrm{P}$ levels may be predictive 
for developing aspiration, silent aspiration and subsequent mortality. Risperidone, which has little influence on serum substance $\mathrm{P}$ productions, is therefore suggested to be a more appropriate first-line drug of choice for treatment of schizophrenia.

\section{Reference}

Arai T, Yasuda Y, Takaya T, et al. 1998. ACE inhibitors and symptomless dysphagia. Lancet, 352:115-16.

Bannon MJ, Lee J-M, Giraud P, et al. 1986. Dopamine antagonist haloperidol decreases substance $\mathrm{P}$, substance $\mathrm{K}$, and preprotachykinin mRNAs in rat striatonigral neurons. J Biol Chem, 261:6640-2.

Chahl LA. 2006. Tachykinins and neuropsychiatric disorders. Curr Drug Targets, 7(8):993-1003.

Elpern EH. 1997. Pulmonary aspiration in hospitalized adults. Nutr Clin Pract, 12:5-13.

Fioritti A, Giaccotto L, Melega V. 1997. Choking incidents among psychiatric patients: Retrospective analysis of thirty-one cases from the West Bologna psychiatric wards. Can J Psychiatry, 42:515-20.

Haleem MA. 1990. Aspiration pneumonia as a cause of death. Br J Clin Prac, 44:398-9.

Holas MA, DePippo KL, Reding MJ. 1994. Aspiration and relative risk of medical complications following stroke. Arch Neurol, 51:1051-3.

Horner J, Massey EW. 1988. Silent aspiration following stroke. Neurology, 38:317-19.

Linden P, Siebens AA. 1983. Dysphagia: predicting laryngeal penetration. Arch Phys Med Rehabil, 64:281-4.

Logemann JA. 1983. Evaluation and treatment of swallowing disorders. San Diego: College Hill Pr.

Nakazawa T, Sekizawa K, Ujiie Y, et al. 1993. Risk of aspiration pneumonia in the elderly. Chest, 103:1636-7.
Nasrallah HA, White T, Nasrallah AT. 2004. Lower mortality in geriatric patients receiving risperidone and olanzapine versus haloperidol: preliminary analysis of retrospective data. Am J Geriatr Psychiatry, 12:437-9.

Pikus L, Levine MS, Yang YX, et al. 2003. Videofluoroscopic studies of swallowing dysfunction and the relative risk of pneumonia. AJR Am J Roentgenol, 180:1613-16.

Pontoppidan H, Beecher H. 1960. Progressive loss of protective relexes in the airway with the advance of age. JAMA, 174:2209-13.

Rabins, PV, Lyketsos CG. 2005. Antipsychotic drugs in dementia: What should be made of the risks? JAMA, 294:1963-5.

Regoli D, Boudon A, Fauchere JL. 1994. Receptors and antagonists for substance P and related peptides. Pharmacol Rev, 46:551-99.

Schneider LS, Dagerman KS, Insel P. 2005. Risk of death with atypical antipsychotic drug treatment for dementia: Meta-analysis of randomized placebo-controlled trials. JAMA, 294:1934-43.

Teramoto S, Matsuse T, Fukuchi Y, et al. 1999. Simple two-step swallowing provocation test for elderly patients with aspiration pneumonia. Lancet, 353:1243.

[FDA] US Food and Drug Administration. 2006. FDA issue public health advisory for antipsychotic drugs used for treatment of behavioral disorders in elderly patients. FDA Talk Paper T05-13. Rockville, MD.

Wang PS, Schneeweiss S, Avorn J, et al. 2005. Risk of death in elderly users of conventional vs. atypical antipsychotic medications. $N$ Engl $J$ Med, 353:2335-41.

Yamaya M, Yanai M, Ohrui T, et al. 2001. Interventions to prevent pneumonia among older adults. $J$ Am Geriatr Soc, 49:85-90.

Zachrisson O, Nomikos GG, Marcus MM, et al. 2000. Effects of antipsychotic drugs on cholecystokinin and preprotachykinin (substance P) mRNA expression in the rat hippocampal formation. Eur Neuropsychopharmacol, 10:355-63. 